\title{
Impact of the gas dynamics on the cluster flux in a magnetron cluster-source: influence of the chamber shape and gas-inlet position.
}

Giuseppe Sanzone a, b, Jinlong Yin a ${ }^{(*)}$, Kevin Cooke ${ }^{a}$, Hailin Sun a, Peter Lievens ${ }^{b}$

a Teer Coatings Ltd, West Stone House, West Stone, Berry Hill Industrial Estate, Droitwich, Worcestershire, WR9 9AS, UK

b Quantum Solid-State Physics, Department of Physics and Astronomy, KU Leuven, B-3001 Leuven, Belgium

(*) Corresponding author: Dr Jinlong Yin (jinlong.yin@teercoatings.co.uk) Teer Coatings Ltd, West Stone House, West Stone, Berry Hill Industrial Estate, Droitwich, Worcestershire, WR9 9AS, UK

\begin{abstract}
Although producing clusters by physical methods offers many benefits, low deposition rates have prevented cluster beam deposition techniques from being adopted more widely. The influence of the gas aerodynamics inside the condensation chamber of a magnetron cluster source on the cluster throughput is reported, leading to an improved understanding of the gas aerodynamics' influence on cluster transport. In the first part of the paper the influence of the carrier gas' inlet position on the cluster flux is studied. In particular, two inlet configurations were investigated, i.e. from the rear of the chamber and from within the magnetron sputtering source. It was found experimentally that the latter configuration can lead to an increased cluster flux, under the same conditions of gas
\end{abstract}


pressure and power applied to the magnetron. This behaviour is explained with the help of simulations. In the second part of the paper, the gas dynamics behaviour inside four chamber shapes, namely two cylindrical shapes with different cross-section diameters and two conical shapes with different apex angles, was simulated. The modelling showed that the fraction of clusters successfully leaving the aggregation zone can be increased by up to 8 times from the worst to the best performing chamber geometries studied. Finally, the cluster throughput was determined experimentally using a quartz microbalance in two of the four chamber designs. It was found that the cluster flux increased up to one order of magnitude, reaching $\sim 20 \mathrm{mg}$ per hour for a condensation chamber with a smaller crosssection and a conical exit.

\section{KEY WORDS}

Clusters, nanoparticles, gas dynamics, gas flow, high flux, wall attachment, and scale-up.

\section{INTRODUCTION}

Although a scientific definition of clusters is still open to debate, they can be considered as aggregates of a discrete number of atoms or molecules (Johnston, 2002). There has been increasing interest in clusters over recent decades since they exhibit unique physical and chemical properties, falling between single atoms/molecules and bulk materials. The range of applications is very wide, extending from biology (Chen, et al., 2018) (Vangala, et al., 2012) (Kumar \& Anthony, 2016), catalysis (Wallace \& Whetten, 2000) (Vajda, et al., 2009), photocatalysis (Sanzone, et al., 2018) (Christopher, et al., 2012) (Simon, et al., 2014) and sensing (Green \& Paget, 2004) to plasmonics (Christopher, et al., 2012) (Linic, 
et al., 2015) (Luk'yanchuk, et al., 2010), photovoltaics (Di Vece, 2019) and spintronics (Serrano-Guisan, et al., 2006) (Zhang, et al., 2009).

Today a vast range of techniques, exploiting both chemical and physical routes, is available to deposit clusters on appropriate supporting surfaces. Chemical methods have the advantage of higher cluster production rate, while physical processes enable improved control of size as well as purity. Among the physical methods, cluster beam deposition by magnetron sputtering and gas-phase aggregation is very popular (Fig. 1)

(Grammatikopoulos, et al., 2016), as it is characterized by a narrow size distribution, easy tunability of cluster size (Fischer, et al., 2015), composition (Vahl, et al., 2017) and structure (Mayoral, et al., 2018), cleanliness of the process (pressure of deposition $\sim 10^{-6}$ mbar), tunability of the cluster-surface interaction by changing the cluster deposition energy (Popok, et al., 2011) (Xirouchaki \& Palmer, 2002), and high deposition rates compared to other standard physical routes (Shyjumon, et al., 2006). Clusters are made from the condensation of a supersaturated vapour, which is generated by eroding a target placed on a magnetron sputtering source. Simplistically, the cluster formation process can be considered as consisting of two steps, the first being three-body collisions between metal atoms and gas molecules, and secondly, atom-by-atom attachment; the second step is deemed much faster than the former (Kashtanov, et al., 2007). Later in the inert-gas condensation process, the coalescence of clusters could also take place (Grammatikopoulos, et al., 2019) if the number density of clusters is sufficiently high. 


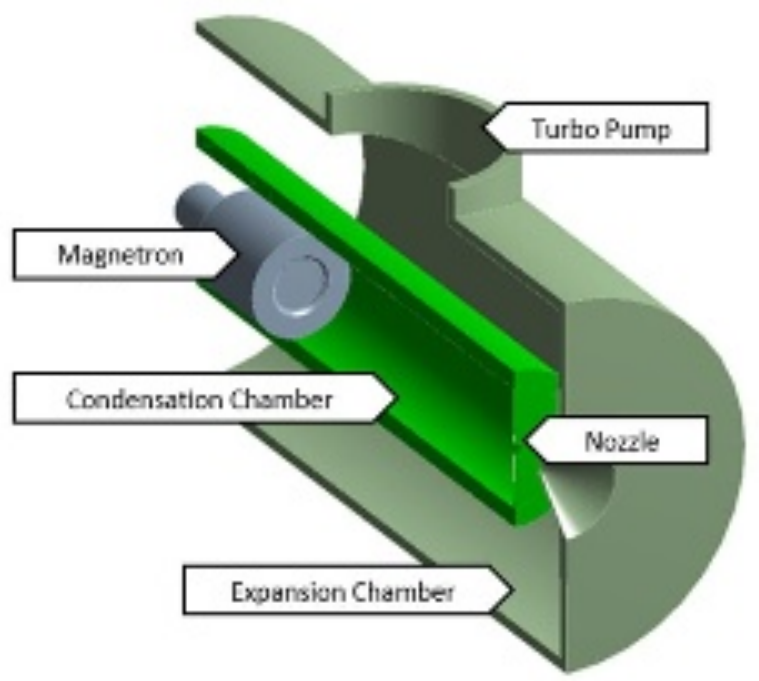

Fig. 1 Cross section view of the cluster source with cylindrical condensation chamber, featuring the magnetron sputtering source, the condensation chamber and the expansion chamber, connected by the nozzle.

A current limiting factor of this technology is the small amount of cluster materials produced, typically of the order of $1 \mu \mathrm{g} \mathrm{hour}^{-1}$, which is only really appropriate for fundamental surface science studies. For many interesting application areas, e.g. model heterogeneous catalysts (Ellis, et al., 2016), the amount of material produced by a state-ofthe-art cluster beam deposition system is still orders of magnitude lower than required. Therefore there is a well-established need in this research field to improve cluster throughput dramatically, facilitating much wider applications, and ultimately the potential for the industrial production of clusters.

An apparatus capable of producing size-selected clusters typically consists of at least three sections: gas aggregation source, ion optics for transmission, and a mass filter for size selection (Hartmann, et al., 2012). All three sections have to be optimised in order to improve the cluster throughput substantially. Several efforts have been reported previously in order to understand the cluster transport in a carrier gas and also the 
focusing of the cluster beam produced via physical methods (Schreiner, et al., 1999)

(Wegner, et al., 2006) (Kousal, et al., 2013). Some groups have studied the influence of the nozzle shape on the gas supersonic expansion and on the cluster axial and radial diffusion in the nozzle region and downstream (Zhang, et al., 2016) (Ganeva, et al., 2014). For the transmission of electrically-charged clusters, B. Elger et al. (Elger, et al., 2017) set up a complete electrostatic model of a cluster deposition apparatus using SIMION, to understand the influence of the electrostatic components on cluster transmission and hence improve the system's efficiency.

Of key importance for such a dramatic increase of the cluster flux is a detailed understanding of the physical mechanisms occurring in the cluster source section, where the metal target is sputtered and clusters are formed in a carrier gas. However, because of the complex nature of the physical mechanisms involved, a rigorous explanation of the clusters' formation and the behaviour of clusters inside the condensation chamber is still incomplete.

In this work, we focus on the cluster generation section only, and investigate how the aerodynamics inside the condensation chamber is affecting the overall cluster-beam throughput. In the first section of the paper, the influence of the gas inlet position on cluster growth and on the cluster flux is investigated. In particular, two configurations of the gas inlet position have been studied: from the rear of the condensation chamber and within the magnetron source (Fig. 2). Although both configurations are used in practice, we are not aware of any comparative studies showing which is more efficient and gives a higher cluster production rate. Our study has combined both aerodynamic simulations and experimental work.

Another important question which has only been partially addressed in the literature, is the chamber geometry's influence on the transportation of the clusters, by influencing the 
gas dynamics inside the condensation chamber. This is important, because of the potential to massively increase the cluster production rate. In this work, we modelled the gas flow inside four different chamber geometries: two cylindrical with a diameter of $100 \mathrm{~mm}$ and $200 \mathrm{~mm}$ respectively, and two conical, both with diameter of $100 \mathrm{~mm}$ but different apex angles, one of $90^{\circ}$ and one of $60^{\circ}$ (Fig. 3). Exploiting critical insights from the simulation results, we constructed two chambers of different geometry (see Fig. S5 in the supplementary document), and experimentally measured and compared the resulting cluster fluxes.

\section{EXPERIMENTAL SETUP AND SIMULATIONS}

\subsection{Experimental}

The cluster-beam apparatus at Teer Coatings Ltd (see Fig. S6) is a Haberland-type magnetron sputtering source, and is described more in detail elsewhere (Ellis, et al., 2016) (Haberland, et al., 1994) (Pratontep, et al., 2005). In this work Ar was used as the inert sputtering gas as well as carrier gas. A DC power supply (MDX500, Advanced Energy) was used to power the magnetron. No cryogenic chamber cooling was used: all the experiments were conducted at ambient temperature. In the expansion chamber a turbomolecular pump with a pumping speed of $1600 \mathrm{l} / \mathrm{s}$ was used. In order to measure the values of the cluster mass flow rate, a quartz crystal microbalance (QCM) was placed in front of the nozzle, $30 \mathrm{~mm}$ away from it. The QCM model is an INFICON Front Load Single crystal sensor, with $6 \mathrm{MHz}$ Au coated crystal and with INFICON STM-2 USB Thin Film Rate/Thickness Monitor. The QCM is mounted on a retractable arm controlled by a linear drive, so that it was possible to simultaneously measure the total cluster flux (with the QCM) as well as the cluster mass spectrum by moving the QCM away from the nozzle axis, hence it no longer obstructs the cluster beam. In this case the charged particles were 
accelerated and focused with a system of Einzel lenses towards a Time of Flight mass spectrometer (ToF) where the cluster mass distribution was measured.

\subsubsection{Gas inlet position}

As shown in Fig. 2, the gas line after the mass flow controller (MFC) was split in two, one sending the gas to the rear of the chamber and the other sending gas to the magnetron source. Each gas line was fitted with a gas valve, enabling control of the gas inlet position inside the condensation chamber throughout the experiments. The desired DC sputtering power was applied to the 2-inch gold sputtering target used for this experiment, while the Ar flow rate was fixed at $150 \mathrm{sccm}$. The aggregation length was varied from $100 \mathrm{~mm}$ to $325 \mathrm{~mm}$ for each gas inlet position.

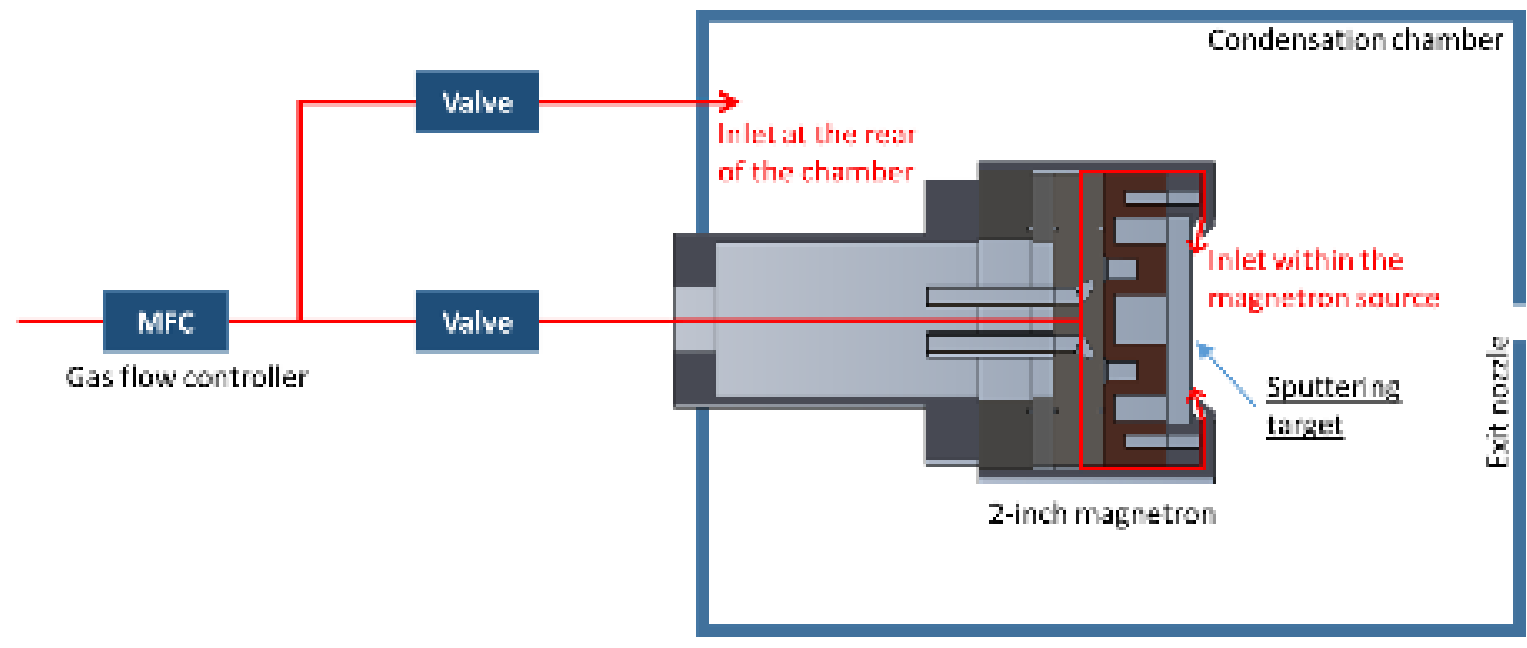

Fig. 2 2-D sketch of the experimental setup involved in this work. After the gas flow controller, a system of two gas lines each provided with a valve allows to control the gas inlet position inside the condensation chamber. The gas can be provided either from the rear of the chamber or from within the magnetron source.

\subsubsection{Condensation chamber geometry}

After simulations on cluster transport had been performed, we experimentally measured and compared the cluster mass fluxes between two chamber designs, namely the optimum-shaped candidate, which was the conical shape with the smaller apex angle of 
$60^{\circ}$, and the conventional cylindrical design with the same internal diameter of $100 \mathrm{~mm}$. The detailed information on the experimental set up of the two geometries can be found in the Section 4 of the Supplementary document.

For cluster flux measurement, the magnetron with a Cu target was sputtered at four different gas flow rates, namely: $60 \mathrm{sccm}, 90 \mathrm{sccm}, 120 \mathrm{sccm}$ and $150 \mathrm{sccm}$. For each gas flow rate, we applied the following DC powers to the 2-inch target: $10 \mathrm{~W}, 20 \mathrm{~W}, 30 \mathrm{~W}, 40$ $\mathrm{W}$ and $50 \mathrm{~W}$.

Furthermore, to ensure that the cluster mass flux measurement was not influenced by previous aggregation conditions, we shut down the power applied to the magnetron and the gas flow controller after each measurement and waited for at least 30 seconds before the next experiment. This allowed metal vapour inside the condensation chamber, in the form of either clusters or single atoms, to be evacuated, so as not to affect the subsequent cluster formation process.

\subsection{Aerodynamic simulation}

In order to quantify and predict the performance of the condensation chamber in terms of cluster flux, a two-step approach was adopted: first the gas dynamics inside the condensation chamber were modelled, and then, after a steady state solution for the gas carrier flow was obtained, discrete particles were released to simulate the cluster transport inside the condensation chamber. In reality, there is also a very small portion of large clusters formed due to cluster coalescence during the late stage of the inert-gas condensation process. However, because of the negligible amount of clusters generated in this way under the chosen conditions, the cluster coalescence process was ignored in the simulation. 
In this study the model for the gas dynamics is pressure-based (Chorin, 1968) and steady state equations were used. Turbulence was taken into account in a realizable $k-\varepsilon$ model (Shih, et al., 1995) (Hinze, 1975) (Reynolds, 1987). All the constants in the model have been used with their default values (Launder \& Spalding, 1974). To prevent any excessive generation of turbulence energy close to stagnation points, a production limiter was used (Menter, 1994). Furthermore, near-wall region effects were taken into account using the enhanced wall treatment for momentum and energy equations (Kader, 1981) (Huang, et al., 1993) (White \& Christoph, 1971). Computational fluid dynamics (CFD) simulations have been performed using ANSYS Fluent 16.1, (ANSYS, Inc.), which solves the NavierStokes equations, treating the gas as a continuous fluid.

Argon is the only gas taken into account, and it has been treated as an ideal gas. This means that $N_{\text {gas }}$ can be extracted from the pressure through the ideal gas law

$$
p=N_{g a s} k_{B} T
$$

Ar specific heat was fixed at $c_{p}=520.64 \mathrm{~J}^{-1} \mathrm{~K}^{-1}$, while for the thermal conductivity and the molecular viscosity values from (Bich, et al., 1990) were used, for temperatures ranging between $90 \mathrm{~K}$ and $290 \mathrm{~K}$. The inlet was set up using the following parameters: mass flow rate $4.5 \times 10^{-6} \mathrm{~kg} \mathrm{~s}^{-1}$ (which corresponds to $150 \mathrm{sccm}$ ), temperature of $290 \mathrm{~K}$, turbulent intensity $13 \%$, hydraulic diameter $1 \mathrm{~mm}$ and supersonic initial gauge pressure $128 \mathrm{~Pa}$. The outlet boundary condition was set up as a pressure-outlet, and the following parameters were used: pressure $0.18 \mathrm{~Pa}$, backflow turbulent intensity $17 \%$, backflow hydraulic diameter $40 \mathrm{~cm}$ and backflow temperature $290 \mathrm{~K}$. The set-up value for the outlet pressure is what we actually read from the vacuum pressure gauge inside the expansion chamber when an Ar flow rate of $150 \mathrm{sccm}$ is provided at the inlet.

After the steady state solution for the gas flow was computed, particle motion was treated in a time-dependent approach taking into account the interaction with the steady state 
solution of the continuous phase (the gas carrier, in this case). The time increment used in our simulations was $0.001 \mathrm{~s}$, and at each time increment 50 particles were released. In this model, the movement of clusters is influenced by two forces, i.e. the drag force and the Brownian force, expressed by (Li \& Ahmadi, 1992)

$$
\frac{d w}{d t}=\frac{36 \mu}{d_{n}^{2}\left(2 \rho_{c l}+\rho_{g a s}\right) C_{c_{n}}}(u-w)+G_{i} \sqrt{\frac{216 \mu k_{B} T}{\pi d_{n}^{5} \rho_{c l}^{2} C_{c_{n}} \Delta t}}
$$

with $w$ and $u$, respectively, the cluster and the gas drift velocity, $\mu$ the molecular viscosity of the gas carrier, $d_{n}$ the cluster diameter, $\rho_{c l}$ and $\rho_{g a s}$ respectively the cluster and gas density, $k_{B}$ the Boltzmann constant, $T$ the cluster temperature, which is assumed to be equal to the gas carrier temperature, $G_{i}$ the zero-mean, unit-variance independent Gaussian random numbers, $\Delta t$ is the time step used in the simulations and $C_{c_{n}}$ the Cunningham factor

$$
C_{c_{n}}=1+\frac{2 \lambda}{d_{n}}\left(1.257+0.4 e^{-1.1 d_{n} / 2 \lambda}\right)
$$

where $\lambda$ represents the gas mean free path. Assuming the number density of clusters $N_{c l}$ is much less than that of the gas carrier $N_{g a s}$, we can write (Sanna \& Tomassetti, 2005)

$$
\lambda=\frac{1}{\sqrt{2} \pi d_{\text {gas }}^{2} N_{\text {gas }}}
$$

where $d_{g a s}$ is the atomic diameter of the gas carrier; in this work the Van der Waals diameter is used.

\subsubsection{Gas inlet position influence}

After taking experimental measurements of the cluster total mass flux, the charged cluster beam current and the cluster mass spectra at different aggregation lengths for both of the gas inlet configurations, simulations were run to model the number density of the 
sputtered metal, $\mathrm{Au}$ in this case, inside the condensation chamber. The sputtered single atoms are treated as discrete particles with a diameter of $0.332 \mathrm{~nm}$ and mass of 196.967 amu. They follow eq. (2), and are introduced in the model from a small portion of the magnetron sputtering target, which corresponds to the experimentally observed racetrack, after the steady state solution for the carrier gas dynamics were solved. For the initial conditions of particle velocity and angular distributions, we used values obtained from simulations of the sputtering process using TRIM (see Supplementary information). As a boundary condition, we assumed that as soon as a particle touches the chamber walls (we consider all the gas domain boundaries as chamber wall, e.g. magnetron head, target etc.) it will be trapped with a probability of 1 . The Au single atom number density spatial distributions for the two inlet configurations are then compared.

\subsubsection{Chamber geometry influence}

In the following discussion, the inlet position is fixed within the magnetron source. To study the influence of the chamber geometry on the cluster flux, two factors are considered, namely, the chamber's internal diameter and the shape of the chamber. For the chamber diameter, aerodynamic simulations were carried out on two cylindrical chambers with an internal diameter of $100 \mathrm{~mm}$, and $200 \mathrm{~mm}$, respectively. For the chamber shape study, two conical chambers with an apex angle of $90^{\circ}$ and $60^{\circ}$ are considered (Fig. 3). 

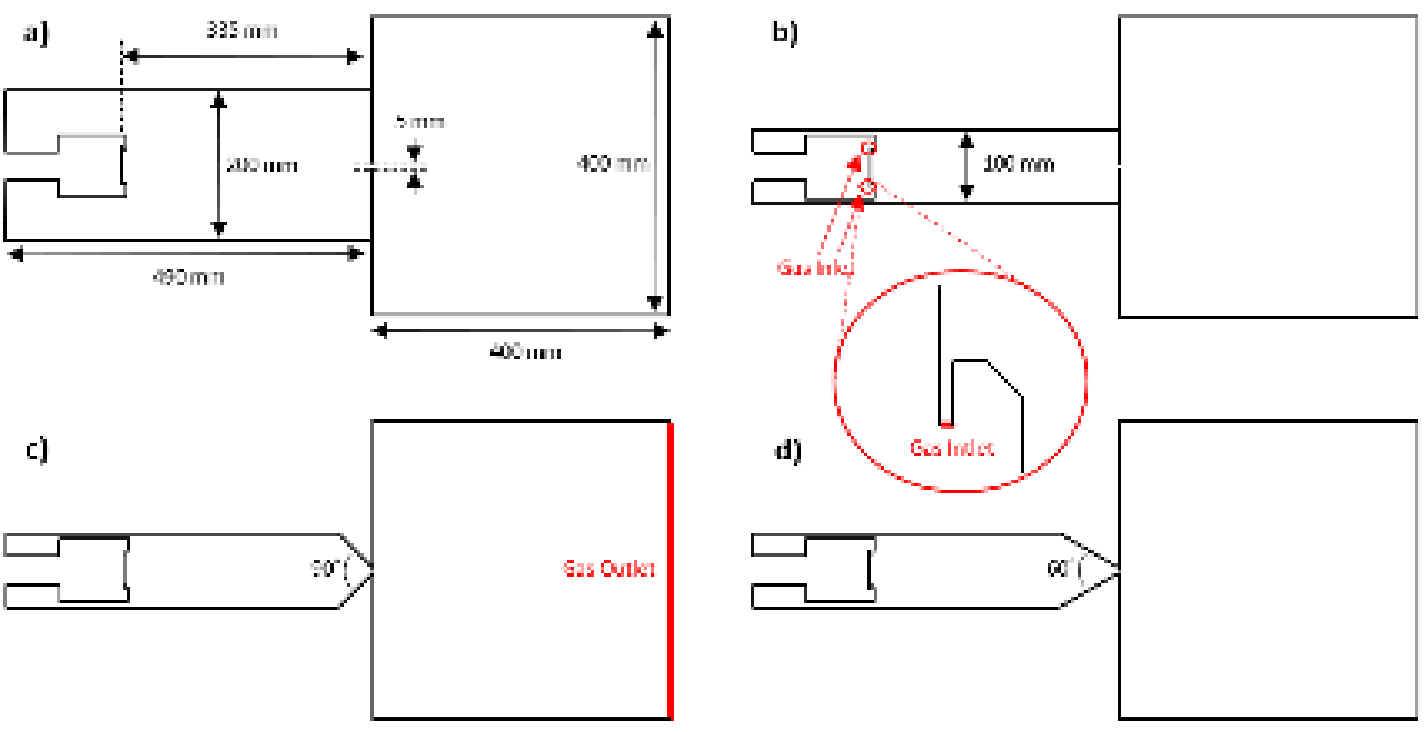

Fig. 3 2-D sketch of the four chamber designs: a) the cylindrical shape with an internal diameter of 200 $\mathrm{mm}$; b) the cylindrical shape with an internal diameter of $100 \mathrm{~mm}$; c) the conical shape with an internal diameter of $100 \mathrm{~mm}$ and an apex angle of $90^{\circ}$; d) the conical shape with an internal diameter of $100 \mathrm{~mm}$ and an apex angle of $60^{\circ}$. The nozzle size was fixed at $5 \mathrm{~mm}$, distance between target and nozzle at $335 \mathrm{~mm}$, condensation chamber length $490 \mathrm{~mm}$, expansion chamber length and diameter 400 mm. Gas inlet and outlet are highlighted in red.

In a similar approach to that described above, after a steady solution is obtained for the gas flow, cluster transport is studied. Clusters with sizes of 10, 100 and 1000 atoms per particle were released from surfaces perpendicular to the axis at several distances from the nozzle, from $10 \mathrm{~mm}$ to $90 \mathrm{~mm}$ in steps of $10 \mathrm{~mm}$. We assume that clusters are small spheres in the framework of a liquid drop model and that their dimensions scale with the Wigner-Seitz radius $r_{W}($ Smirnov, 2012)

$$
n=\frac{4 \pi \rho_{b u l k}}{3 m} r_{n}^{3}=\left(\frac{r_{n}}{r_{W}}\right)^{3}
$$

$\rho_{\text {bulk }}$ being the density of the corresponding bulk material, $m$ the atomic mass and $r_{n}$ the cluster radius. In this framework, also, the density of the cluster matches the density of the 
corresponding bulk material, i.e. $\rho_{c l}=\rho_{\text {bulk }}$. In this case the standard values for copper present in the software database were used.

It should be noted that by releasing particles with different sizes at different distances from the nozzle, we are not assuming that these clusters are formed at these positions. The clusters could be formed there, or elsewhere. The key characteristic of interest is the percentage of clusters having a chance to leave the chamber, for a given number of clusters with a specified size and released at such positions. The cluster formation and growth processes have been studied by several groups, e.g. (Mattei, et al., 2019) (Kousal, et al., 2018), and were found to be influenced by the geometry of the chamber, target dimensions, inlet position, gas flow rate, gas temperature, species involved and power applied to the magnetron. The study of such processes goes beyond the scope of this work. Here, our aim is to calculate the probability of clusters with different sizes successfully leaving through the nozzle as a function of the distance that they need to travel, and then to compare these probabilities for the different chamber geometries studied.

In the aerodynamic modelling software, cylindrical symmetry was applied to the model of the condensation chamber in order to reduce the calculation time. For all the simulated results in this work, only half of the chamber was shown, the other half being symmetric with respect to the horizontal axis.

\section{RESULTS AND DISCUSSION}

\subsection{Influence of the gas inlet position}

Fig. 4 shows QCM measurements of the mass flux of Au clusters (and single Au atoms) leaving the condensation chamber, versus the aggregation length, i.e. the distance between the sputtering target and the nozzle. The Ar flow rate was fixed at $150 \mathrm{sccm}$, and $8 \mathrm{~W}$ of 
power was applied on the magnetron. Results are compared for the two gas inlet configurations: within the magnetron source and at the rear of the chamber.

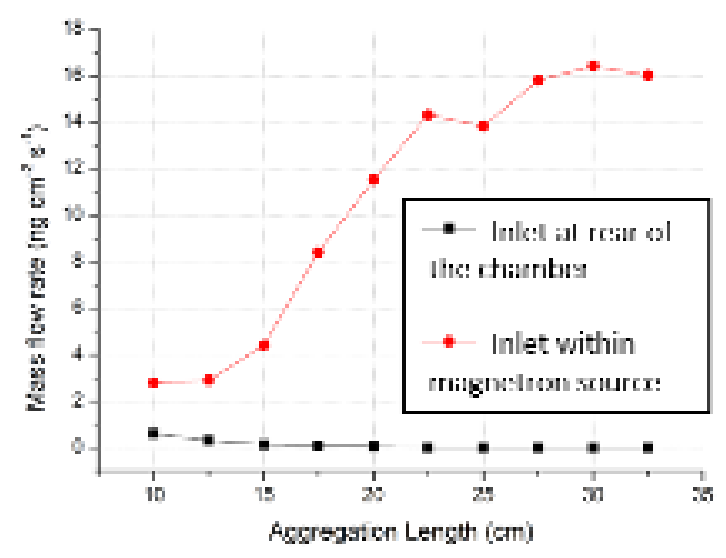

Fig. 4 Mass flow rate of $\mathrm{Au}$ atoms/clusters at different aggregation lengths, when the gas inlet position is within the magnetron source (red circles) or at the rear of -the chamber (black squares). The Ar flow rate is fixed at $150 \mathrm{sccm}$, and the sputtering power is maintained at $8 \mathrm{~W}$.

It is well known that the flux of Au single atoms sputtered out of the target is constant when both sputtering power and gas flow rate are kept at their fixed values. It might be assumed that the material flux coming out of the condensation chamber would also be constant, however, this is not the case as can be seen in Fig. 4; in fact, the mass flux varies with the aggregation length. To explain this, the two competing forces on particles travelling inside the condensation chamber should be considered. The drag force pushes the particle to follow the gas streamlines while the Brownian force causes the particle to diffuse randomly in any possible direction. From eq. (2) it can be deduced that, for a particle, the ratio between the drag force and the Brownian force increases with the square root of the particle size, i.e. Drag/Brownian $\propto \sqrt{d_{n}}$. This means that the smaller the particle, the higher the probability that it will diffuse away from the gas streamlines.

In the case of the gas coming through the magnetron source itself, when the sputtering target is at its closest distance to the nozzle in our experimental configuration, i.e. $100 \mathrm{~mm}$ 
of aggregation length, the QCM starts recording a tiny amount of mass flow rate corresponding to $\sim 3 \mathrm{ng} \mathrm{cm}^{-2} \mathrm{~s}^{-1}$. At this short distance, the majority of material leaving the aggregation zone through the nozzle is in the form of single atoms, as confirmed by the mass spectra shown in the supplementary information (Fig. S1). Single atoms have the smallest possible particle diameter, hence they are hardly influenced by the drag force and their movement is dominated by Brownian motion. This random movement means they do not exhibit a preferred direction of motion (for example, the direction of gas streamline), and they diffuse freely in the condensation chamber until they land somewhere or escape the chamber. As a result, the majority of single atoms are deposited onto the chamber walls, and only a small fraction escape through the nozzle by chance, to be detected by QCM.

When the aggregation length is increased, the residence time of a particle inside the aggregation zone increases as well. This enhances the probability for such a particle to collide with other particles, giving rise to larger particles which are less affected by Brownian diffusion. This is the common phenomenon people have observed and reported that greater cluster size is correlated with the increase of aggregation length. In this process, there are increasing number of single Au atoms integrated into Au clusters, and correspondingly fewer of them deposit on the chamber wall. For the resulting Au clusters, they are more affected by the drag force and have a much higher chance to escape from the condensation chamber. This explains why the mass flux measured by the QCM increases monotonically with increased aggregation length.

When the gas is provided from the rear of the chamber, it is clear from Fig. 4 that the mass flow rate gradually decreases to zero when the aggregation length is increased. This seems to suggest that only single gold atoms were produced and very few or no clusters were formed. As the magnetron source is slowly moved away from the exit nozzle, the 
probability for single Au atoms diffusing from the magnetron to the position of the nozzle is consistently reducing because of the nature of Brownian motion. At an aggregation distance of $300 \mathrm{~mm}$, this probability has dropped to almost zero. The mass spectra also confirm that there is no cluster formation under such conditions (see Supplementary Information Fig. S2).

It is suggested that a higher number density of Au atoms would enhance the cluster nucleation process, which should then lead to the formation of more clusters. An effective way to increase the number density of metal atoms is to increase the sputtering power. In the following experiment, a DC sputtering power of up to $31 \mathrm{~W}$ was applied to the magnetron, while the aggregation length is fixed at $275 \mathrm{~mm}$ to give ample opportunity for clusters to grow. The measured results for the mass flux detected on the QCM is summarised in Fig. 5.

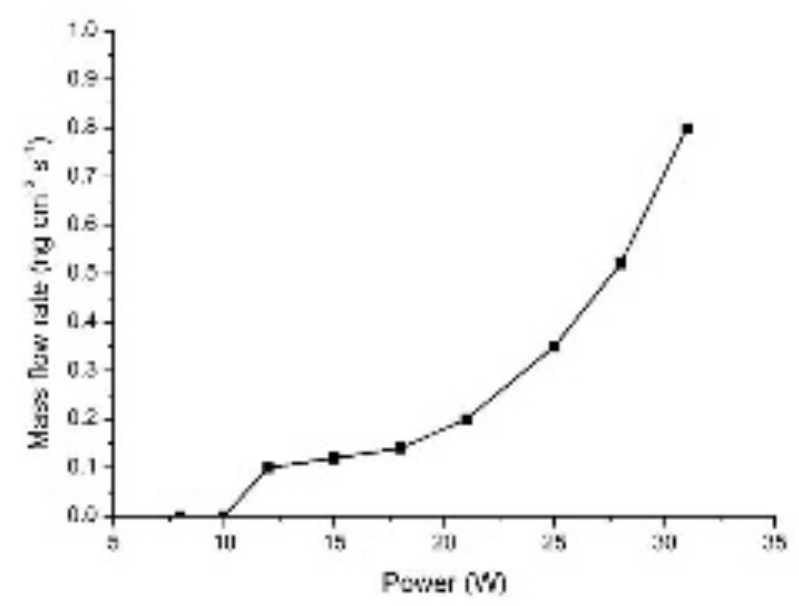

Fig. 5 Mass flow rate of Au clusters at different sputtering power, when the gas inlet position is at the rear of the chamber. The aggregation length is fixed at $275 \mathrm{~mm}$, and the gas flow rate is kept at 150 sccm.

At such distance the probability for single atoms to successfully leave through the nozzle is dramatically reduced because of their high Brownian diffusivity. Therefore nearly all the 
mass flux signals from the QCM could be attributed to agglomerates of atoms, i.e. clusters. As shown in Fig. 5, there is no signal detected when the DC sputtering power is in the range between $6 \mathrm{~W}$ and $10 \mathrm{~W}$. However, when the power is increased to $12 \mathrm{~W}$, we start to see a small mass flux of $\sim 0.1 \mathrm{ng} \mathrm{cm}^{-2} \mathrm{~s}^{-1}$, and then this signal steadily grows with increasing applied power until the experiment is terminated at $31 \mathrm{~W}$. The resulting cluster size distribution in this case is shown in Fig. S3 of the Supplementary Information.

These results confirmed that Au clusters indeed can be produced when the gas inlet is at the rear of the chamber; however, the cluster mass flow rate is more than 20 times lower, $\sim 0.8 \mathrm{ng} \mathrm{cm}^{-2} \mathrm{~s}^{-1}$ versus $\sim 16 \mathrm{ng} \mathrm{cm}^{-2} \mathrm{~s}^{-1}$ when gas inlet within the magnetron source (Fig. 4), even though a much higher sputtering power was applied (almost 4 times, $31 \mathrm{~W}$ vs. 8 W). The cluster formation process seems to be very inefficient in this case. To investigate the potential causes of this significant difference in cluster growth, gas flow dynamics simulations were carried out.

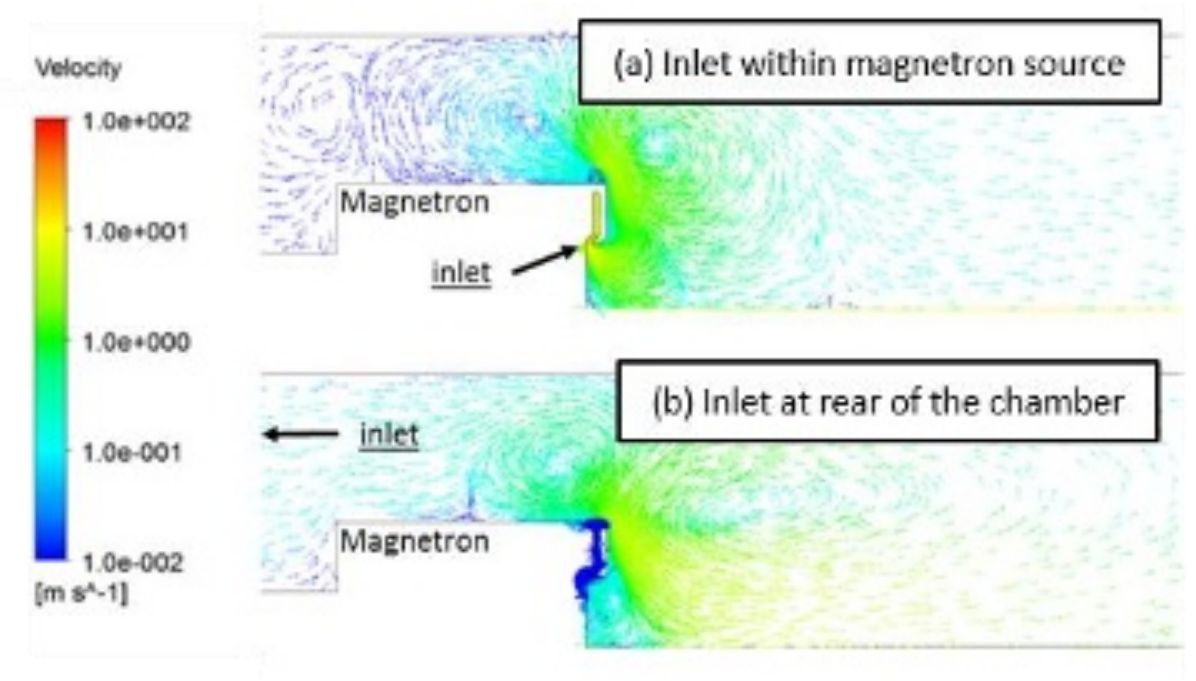

Fig. 6 Ar gas velocity profiles, $150 \mathrm{sccm}$ flow rate at room temperature, (a) when gas enters the chamber through the inlet within the magnetron source; (b) when gas enters through the inlet at the rear of the chamber. Only top half of the condensation chamber is shown here, as the bottom half is symmetrical along the horizontal axis. 
Fig. 6 shows the velocity profiles of the carrier gas Ar. The arrows indicate the flow direction, and the colour goes logarithmically from blue, which represents low velocity $\left(v \leq 0.01 \mathrm{~m} \mathrm{~s}^{-1}\right)$, to red, which represents high velocity $\left(v \geq 100 \mathrm{~m} \mathrm{~s}^{-1}\right)$. Overall, the gas velocity profiles for both gas inlet configurations are very similar. The greatest difference is found in the region in front of the magnetron target. In this region, the gas velocity is in the range from a few m/s to $10 \mathrm{~m} / \mathrm{s}$ for gas inlet within the magnetron source (Fig. 6a), while it is about 10 times lower at around $0.1 \mathrm{~m} / \mathrm{s}$ for gas inlet at the rear of the chamber (Fig. 6b). Because the drag force is directly proportionally to the gas velocity (Eq. 2), it is very likely that the stronger drag force in front of the sputtering target with gas inlet next to it would reduce the possibility of redeposition of the sputtered species back to the target. In a typical magnetron sputtering process, there always is a portion of sputtered atoms deposited back to the cathode (i.e. target), and sometimes this portion can be as high as $40 \%$, depending on many factors (Rossnagel, 1988). If more sputtered atoms were redeposited onto the target, there would be fewer single atoms available for cluster formation. This could explain the dramatic mass flow rate reduction observed when the gas inlet was moved to the rear of the chamber.

To further investigate this hypothesis, we carried out aerodynamic simulations to track the movement of single atoms in this region (see Section 2.2.1). After the gas velocity profiles (Fig. 6) were calculated, packets of particles with the transport properties of $\mathrm{Au}$ single atoms were released from a small ring-shaped area on the target, which corresponds to the actual eroded racetrack experimentally measured on our target. Because of the difference in gas velocity profiles between the two gas inlet configurations, the released particles exhibited different spatial distribution in terms of number density of $\mathrm{Au}$ single atoms mainly for two reasons. Firstly, a lower gas velocity close to the sputtering target surface means that the sputtered atoms would experience less drag force from the carrier gas. Under such conditions, the probability for single atoms to travel back and 
deposit on the target surface is higher. Indeed, from simulation results, it was possible to calculate the fraction of sputtered atoms redepositing onto the target surface. We found that such fractions amount to $76 \%$ and $68 \%$ respectively for the configuration with the gas inlet from the rear of the chamber and from within the magnetron source. This means that, if the gas is provided from within the magnetron, the effective amount of sputtered atoms available in the gas phase for cluster formation and growth is $\sim 1.3$ times higher than that obtained if the gas inlet is at the rear of the chamber. Secondly, the single atoms which are found in the gas phase distribute differently for the two inlet configurations. Fig. 7a shows the Au single atom number density distribution inside the condensation chamber. Here a logarithmic scale is used to better indicate how single atoms would be more evenly distributed across the chamber if the gas was provided from the rear. On the other hand, when the gas inlet was within the magnetron source, atoms would be more localized in a central region of the chamber, closer to the target surface. Fig. $7 \mathrm{~b}$ shows the single atom number density in a region close to the sputtering target surface, here using a linear scale to highlight the difference between the two gas inlet configurations investigated. It is easy to see how the gas inlet within the magnetron source would better localize the single atoms, providing higher densities of the sputtered species.

It is generally accepted that the three-body collision process, involving the collision of two metal atoms and one gas molecule at the same time, is how a dimer cluster is formed. This is a critical step, which paves the way for the subsequent cluster growth. Ideally, we would like to calculate the probability of a three-body-collision in front of the sputtering target for each case. However, this is not currently feasible due to available software limitations. Instead, the Au atom-atom collision probability, versus the distance from the sputtering target, is calculated as shown in Fig. 7c, based on the principle that such probability is proportional to the square of single atom number density (Sanna \& Tomassetti, 2005). It is suggested that the probability of metal atom-atom collisions is proportional to the 
probability of three-body collision. A high probability of metal atom-atom collision should lead to a high probability of three-body collision, especially when the number density of gas molecules (Ar) is high in the system (a typical ratio between Ar molecule and metal atom number density is around 1000:1). From Fig. 7c, we can see that when the gas is sent in through the magnetron source, the Au atom-atom collision is much more probable, and the high probability also spans a much larger region, compared to the case when gas is introduced from the rear of the chamber. All this evidence is consistent with more efficient cluster formation and growth in the case of the magnetron source inlet, and hence in line with the higher mass flow rate measured on the QCM.

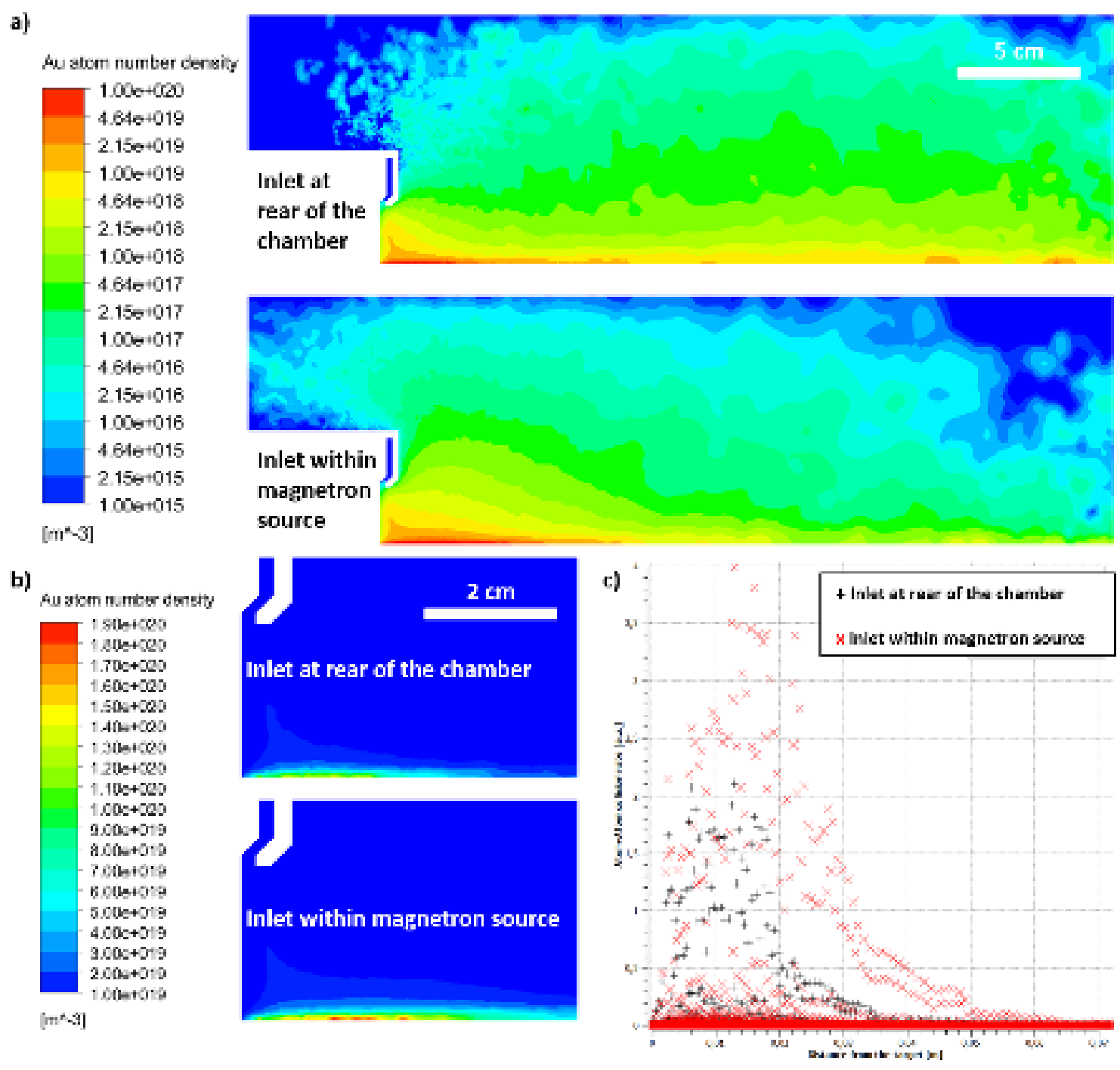


Fig. 7 a) Au single atom number density profile inside the condensation chamber in the case the gas is provided from the rear of the chamber (top) and from within the magnetron source (bottom). A logarithmic scale is used to show the number density distribution. b) Au single atom number density profile in a region close to the target surface, in the case the gas is provided from the rear of the chamber (top) and from within the magnetron source (bottom). A linear scale is used to show the number density distribution. c) The probability of Au atom-atom collision in front of the sputtering target, if the gas inlet is within the magnetron source (red cross symbol) and at the rear of the chamber (grey dash symbol).

In summary, to design a high-rate cluster production instrument, the carrier gas should be injected into the condensation chamber at a place as close to the sputtering target surface as possible, which brings two key benefits, i) reduced redeposition of sputtered atoms onto the sputtering target; and ii) increased atom-atom collision probability.

\subsection{Effects of the chamber geometry}

The previous section showed how a small change in the position of the gas inlet could result in a large variation in the measured cluster flux. This clearly demonstrates the important role of gas dynamics in the process of cluster nucleation and formation. In the condensation chamber, when the Ar flow rate is around $150 \mathrm{sccm}$ and the exit nozzle size is $5 \mathrm{~mm}$, the measured gas pressure is at $\sim 80 \mathrm{~Pa}$. This gives a mean free path $(\lambda)$ of $\sim 1$

mm for the Ar gas molecules. The calculated Knudsen number $K_{n}=\frac{\lambda}{L} \sim 0.01$ (where $L=$ $100 \mathrm{~mm}$ is the internal diameter of the condensation chamber), indicates that the gas stream can be treated as continuum flow.

The nucleation and formation of clusters is a very complicated process, influenced by many macroscopic parameters, and it has been investigated by other groups, e.g. Khojasteh et al. (Khojasteh \& Kresin, 2017). Here we focus the discussion on the transportation of clusters from where they are formed (which could be anywhere inside the chamber) to the position of the exit nozzle where they leave the condensation 
chamber. As shown in Fig. 6, the gas drift velocity is at its highest along the central axis of the condensation chamber, and gradually reduces to nearly zero when approaching the chamber wall. It can be imagined that, in this scenario, differently sized clusters and single metal atoms diffuse, frequently colliding with gas molecules or among themselves.

Because single atoms are dominated by Brownian motion, they are distributed more widely in the chamber. As for the large clusters, they are more likely to be found along the central axis of the chamber where the gas drift velocity is highest. This is because the movement of large clusters is influenced more significantly by the drag force exerted on them by the carrier gas. For medium sized clusters, it is reasonable to expect behaviour somewhere between the two cases of single atoms and large clusters.

Because of the random movements of single metal atoms and of very small clusters containing a few atoms, it is not expected that any optimised aerodynamic design would significantly improve the transportation efficiency of such species to the position of the exit nozzle. However, we might be able to dramatically increase the mass flux of mediumsize and large clusters by efficiently transporting them out of the chamber with an optimised novel aerodynamic design. There are several approaches to optimise the gas dynamics, for example, changing the gas flow rate, changing the chamber geometry, adding ridges on the chamber wall, or drilling holes on the chamber wall, etc.

However, changing gas flow rate is not a feasible approach in practice. To produce clusters, the pressure inside the condensation chamber has to be maintained in a narrow range. For too high a pressure ( $>100 \mathrm{~Pa}$, typically), the magnetron sputtering process would fail, mostly because of internal discharge and arcing between cathode and anode; if too low $(<10 \mathrm{~Pa})$, clusters will not be formed because of the low collision rate due to their increased mean free path. Although adding ridges or drilling holes on the chamber wall would definitely bring a considerable change to the gas dynamics, we decided to 
investigate the influence of chamber diameter and chamber shape on the aerodynamics of carrier gas. As similar research has not been reported previously, we start from the basics and develop some conclusions which could inform the future development of more complex conceptual designs (e.g. ridges or holes, etc.).

Two cylindrical chamber designs are compared, with 100 and $200 \mathrm{~mm}$ internal diameter. In addition, two conical chambers are also compared, with $60^{\circ}$ and $90^{\circ}$ apex angle. In all four cases, the gas inlet position is fixed within the magnetron source for both simulation and experimental work.

Considering the fate of a cluster generated inside the chamber, it either diffuses to the low gas velocity region close to the chamber wall and lands there; or a cluster may diffuse to the high velocity region along the central axis of the chamber, follow the drifting carrier gas and leave the chamber through the exit nozzle. To improve the cluster transportation efficiency, the aim is to minimise the possibility of clusters' attachment to the walls, and maximise the possibility of clusters leaving the chamber. From eq. (2) we can see that, for a particle moving inside a carrier gas, the drag force is proportional to the gas velocity $u$. This means that in order to minimise the probability for the clusters to diffuse to the chamber walls, we need to maximise $u$, especially in the area close to the chamber walls where any excessive particle diffusion would increase the chance of clusters landing there. Fig. 8 shows the simulated results of the gas drift velocity profile in the four differently shaped chambers. Similar to the labels in Fig. 6, the arrows indicate the flow direction, and the colour goes logarithmically from blue, which represents low velocity $\left(v \leq 0.01 \mathrm{~m} \mathrm{~s}^{-1}\right)$, to red, which represents high velocity $\left(v \geq 100 \mathrm{~m} \mathrm{~s}^{-1}\right)$. It can be seen in Fig. 8 a that, in the case of a cylindrical chamber with cross-sectional diameter of $200 \mathrm{~mm}$, some eddies develop at the top-right corner of the chamber. In this region, the Brownian diffusion forces would prevail over the drag force (Li \& Ahmadi, 1992), especially for smaller 
clusters. When the diameter is decreased by half (Fig. 8b), the overall velocity profile increases in magnitude. This is easily explained by the law of the mass conservation, i.e. $\rho$. $A \cdot u$ must be constant, with $A$, in this case, being the chamber cross-sectional area. If $\rho$ does not change, which is true for all the cases investigated, the product $A \cdot u$ must be conserved, which means that decreasing the diameter $d$ would increase the average gas drift velocity $u$, i.e. $u \propto d^{-2}$. Furthermore, when the diameter is decreased, the eddies near the top-right corner become smaller (Fig. 8b). In the case of the conical shape (Fig. 8c) the eddies are completely removed, the gas drift velocity close to the wall increases in the direction of the chamber axis, and this effect is enhanced further if the apex angle is reduced (Fig. $8 \mathrm{~d})$.

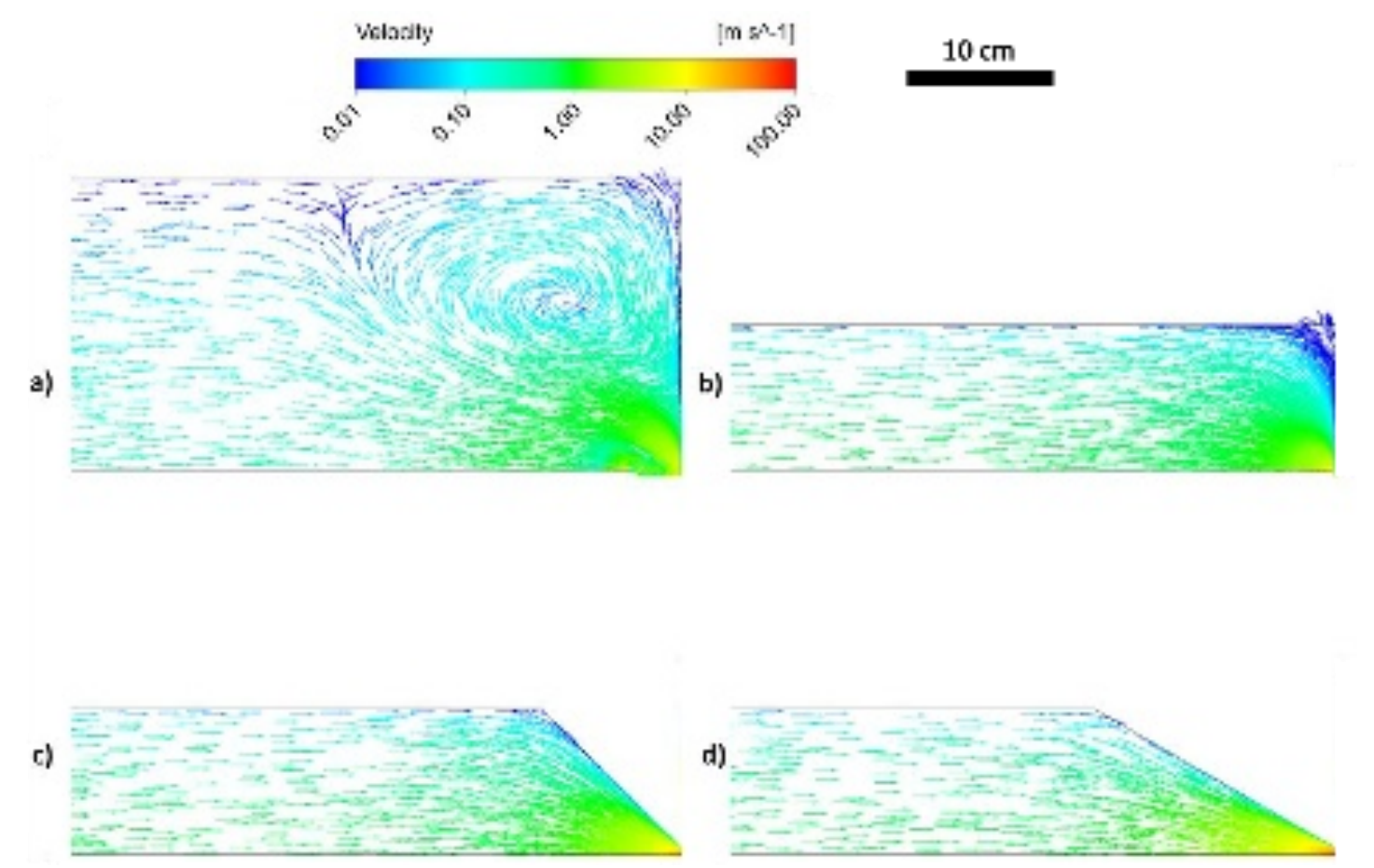

Fig. 8 Gas velocity profile inside the condensation chamber. The arrows denote the local gas velocity direction, the colour show in a logarithmic scale the velocity magnitude, from blue for a few centimetres per second to red for $\mathbf{1 0 0}$ meters per second. The gas velocity profiles are shown for the four chamber geometries investigated: a) cylindrical with diameter of $200 \mathrm{~mm}$; b) cylindrical with diameter of $100 \mathrm{~mm}$; c) conical with apex angle of $90^{\circ}$; d) conical with apex angle of $60^{\circ}$. 
In order to compare the performance in terms of cluster flux among the differently shaped chambers, copper clusters with sizes of 10,100 and 1000 atoms per particle were released from multiple vertical lines perpendicular to the central axis of the chamber. The first vertical line is selected at a position $10 \mathrm{~mm}$ away from the exit nozzle; with an equal spacing of $10 \mathrm{~mm}$ between the lines, the furthest line is $90 \mathrm{~mm}$ away. As the spatial distribution of clusters inside the condensation chamber is unknown, a simple approach is taken for the simulation in which the number of $\mathrm{Cu}$ clusters are evenly distributed along the lines. Inevitably, this simplified approach limits the accuracy of the simulated results; but, we believe the overall trends exposed in the simulation results should give some insights on the aerodynamic influence on the transportation of particles.

To quantify the probability of particles coming out of the condensation chamber, the number of particles going through the exit nozzle were counted. In Fig. 9 the fraction $f$, between the number of particles passing through the nozzle and the number of particles released at a given line, is plotted against the distance between this line and the nozzle. It should be noted that in the case of 1000 atoms per cluster, the simulation has not been carried out for all the distances, since this would have required excessively long computation time. 

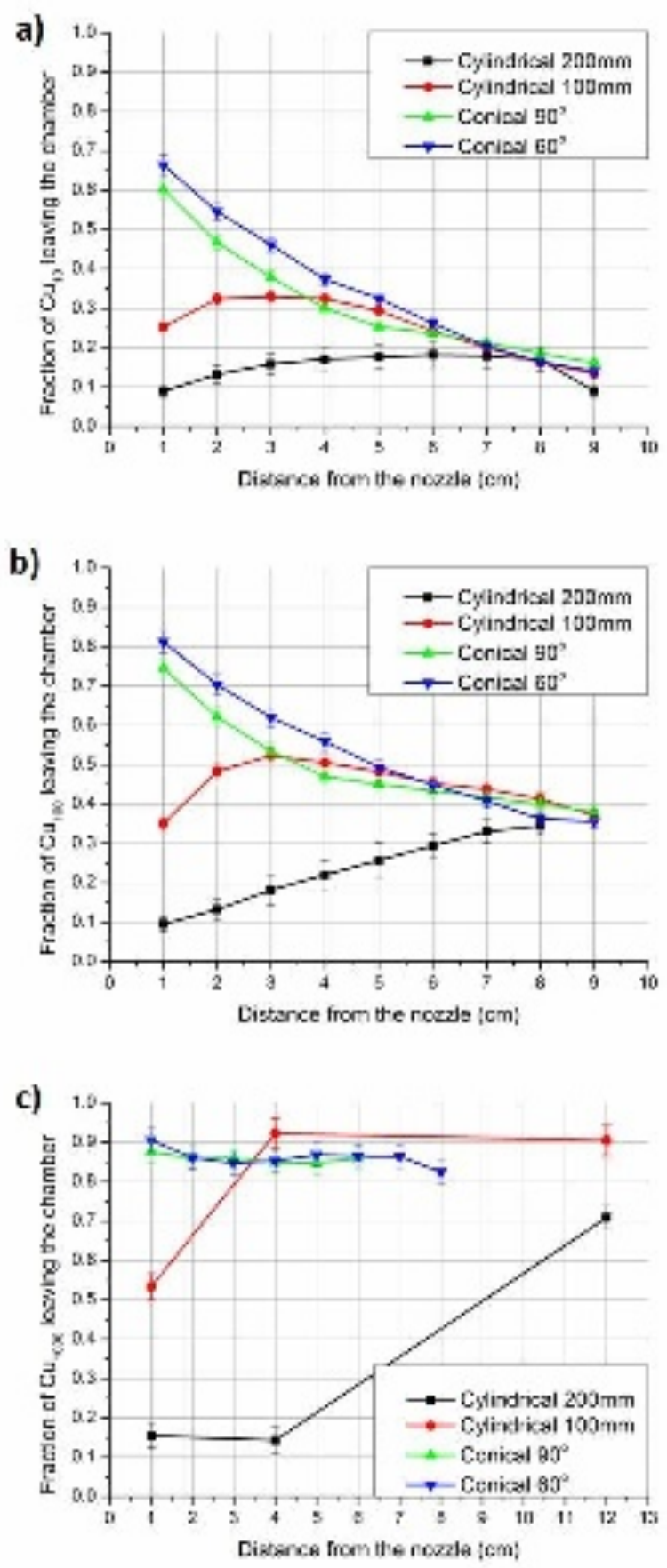

Fig. 9 Fraction of clusters departing from lines perpendicular to the chamber axis and successfully going out through the nozzle versus the distance of such lines from the nozzle. Results for clusters of different sizes are shown here: a) 10 atoms per cluster; b) 100 atoms per cluster; c) 1000 atoms per cluster. 
It is clear that for all of the chamber geometries simulated, the fraction $f$ which actually can be treated as the probability of clusters coming out of the chamber, increases as the distance to the nozzle is reduced. This is not surprising and is readily explained. As the $\mathrm{Cu}$ clusters are released from the lines closer and closer to the nozzle, they would have less chance to diffuse to the chamber walls and deposit there. As a result, increasing numbers of particles should leave the chamber.

Large differences are apparent in the fractions $f$ among the four chamber geometries simulated, especially at shorter distance to the nozzle. The general trend is that the smaller the chamber diameter, the higher the fraction $f$; and similarly, the smaller the apex angle of the conical chamber, the higher the fraction $f$. We attribute this behaviour to the influence of gas drift velocity $u$. As mentioned earlier, higher gas velocity gives rise to stronger drag force, which would push more particles to travel in the direction of gas streamline. As seen in Fig. 8, among the four simulated chamber geometries, the 100-mm conical chamber with an apex angle of $60^{\circ}$ has the highest gas velocity in the proximity of nozzle. This explains why this geometry always gives the highest fraction $f$.

On the other hand, if the $\mathrm{Cu}$ particles are released at a distance further away from the nozzle, say $8 \mathrm{~cm}$ or $9 \mathrm{~cm}$ or greater, there is virtually no difference in the resulting fractions $f$ between chamber geometries. This implies that if clusters stop growing (or the cluster size distribution is stable) at large enough distances from the nozzle, we would not be able to appreciate any difference in terms of cluster flux between the chambers with different geometry. However, we are confident that this is rarely relevant. In fact, the results shown in the Supplementary information Fig. S1 provide a clear example that clusters can continue to grow, even after the aggregation length is increased to $325 \mathrm{~mm}$.

Another interesting result is that the larger the cluster, the higher the chance that it can escape the chamber. For example, in the case of $100-\mathrm{mm}$ conical chamber with $60^{\circ}$ apex 
angle, the Cu10 clusters have a probability of $66 \%$ of exiting the chamber if they are released $10 \mathrm{~mm}$ away from the nozzle. The chance is increased to $82 \%$ for Cu100 clusters, and then $91 \%$ for Cu1000 clusters. Obviously, larger clusters are affected more by the drag force, and less influenced by Brownian force. As a result, they tend to travel in the direction of carrier gas and leave the chamber along with it.

Finally, we verified the influence of the chamber geometry by measuring the cluster mass flux with a quartz crystal microbalance (QCM) for different configurations. Because of the high manufacturing cost involved, we only built and tested two designs of chamber, a "standard" cylindrical chamber and a conical shape design with an apex angle of $60^{\circ}$, both of them with an internal diameter of $100 \mathrm{~mm}$ (Fig. S5). Fig. 10 shows the experimental results of mass flux measured for a 2 -inch $\mathrm{Cu}$ target $335 \mathrm{~mm}$ away from the nozzle (the exact conditions simulated in the model), sputtering at $10 \mathrm{~W}, 20 \mathrm{~W}, 30 \mathrm{~W}, 40 \mathrm{~W}$ and $50 \mathrm{~W}$ power under different Ar flow rate conditions (60 to $150 \mathrm{sccm}$ ).

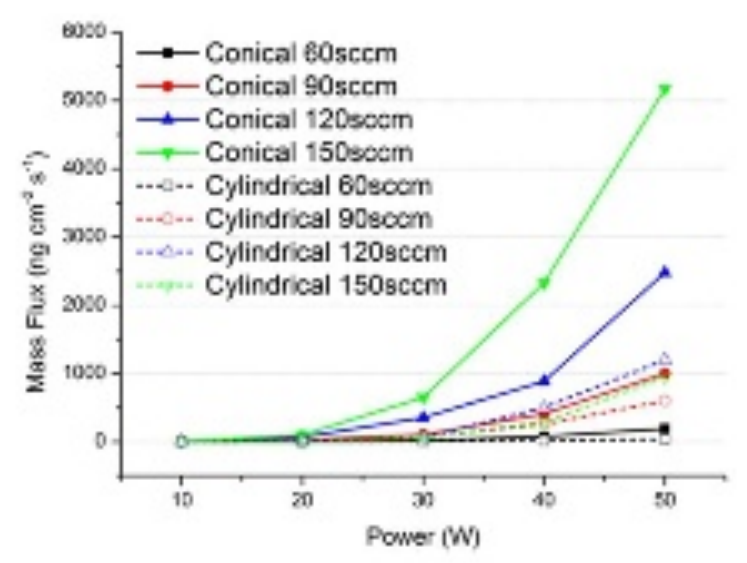

Fig. 10 Experimental measurements of the cluster mass flux at a distance of $30 \mathrm{~mm}$ from the nozzle. Continuous lines are the results for the conical shape chamber with an apex angle of $60^{\circ}$, dashed lines are for the cylindrical shape.

Initially we observed the mass flux increases with the DC power applied to the magnetron. This is in agreement with results presented in Fig. 5, where the gas is provided from the 
rear of the chamber, suggesting that clusters form in the same way for both of the gas inlet configurations studied. Basically, an increment in the power applied means an increase of the number density of the sputtered species and therefore the number of clusters formed. It is also shown in Fig. 10 that the increase of the gas flow rate would enhance the overall cluster flux, which is in agreement with theoretical results already provided by other groups, see e.g. (Smirnov, 2012).

Secondly, it should be noted that for the conical chamber we observed a systematic increase in the resulting cluster flux for all the values of power and gas flow rate applied, compared to the cylindrical chamber. However, there are some clear discrepancies between the experimental and simulated results. The QCM measurements show an increase of the cluster flux up to ten times, while in the calculation a maximum increase of three times is indicated. There are two possible reasons for such discrepancy. Firstly, in the numerical simulation model, a uniform particle number density distribution along the release lines has been assumed, which causes an error. Secondly, as the clusters are moving in the conical chamber, they would be increasingly constrained into a smaller volume as they approach the nozzle. This would increase the local cluster number density. This increased number density would enhance the coalescence processes between particles, hence increasing their probability of leaving the condensation chamber. Nevertheless, these data clearly show that the configuration with the conical shape outperforms the cylindrical one for all the values of Ar flow rate and sputtering power applied to the target, indeed up to ten times better under the selected conditions.

We expect that the cluster flux could be further enhanced if the apex angle of the conical chamber is reduced from $60^{\circ}$, as a higher portion of clusters would leave the condensation chamber instead of attaching to the chamber wall. If the apex angle is small enough, the cylindrical section of the chamber would disappear, and we would have a pure conical 
chamber. It is estimated that the pure conical geometry could increase the cluster flux by up to 5 times when compared to the current $60^{\circ}$ conical geometry, depending on the cluster size. When comparing to a standard cylindrical geometry, this would represent a cluster throughput increase of nearly 50 times, approaching the requirements of a potential industrial application of nanoclusters, for example, as a high-performance catalyst for a niche high added-value catalysis chemical market.

\section{CONCLUSIONS}

In this work we have demonstrated how the gas dynamics inside the condensation chamber of a Haberland-type cluster-beam system can significantly affect the cluster throughput, in particular how the gas inlet position has an impact on the cluster formation and how the chamber shape affects the cluster mobility. Supported by simulations of the transport of the sputtered atoms, we showed that if the gas is provided through the magnetron source itself it helps in reducing redeposition of sputtered atoms on the magnetron target, and consequently enhancing the resulting cluster formation and growth. For the other inlet configuration where the gas is introduced from the rear of the chamber, it was confirmed that clusters can be produced and similar cluster flux could be obtained, though requiring a much higher magnetron sputtering power because of the correspondingly low efficiency in cluster formation.

In addition, we presented modelling results for four different geometries of condensation chamber: two "standard" cylindrical shapes with cross section diameter of $200 \mathrm{~mm}$ and $100 \mathrm{~mm}$, and two conical shapes, both with diameter of $100 \mathrm{~mm}$, one with apex angle of $90^{\circ}$ and the other one with apex angle of $60^{\circ}$. These simulations indicated, theoretically, that the conical design with smaller apex angle is the most efficient. By reducing the chamber diameter and reducing the apex angle for conical chamber, the gas velocity 
profile was optimised in such a way that the gas velocity close to the walls was increased. As a result, the probability of attachment of clusters to the walls (and hence lost from the cluster generation process) was reduced. We also experimentally proved that the new design performs several times better than the standard cylindrical design. This work has also shown that the detailed understanding of the aerodynamics of cluster production systems is important and should be exploited in order to further progress the scale-up of cluster deposition techniques.

\section{SUPPLEMENTARY MATERIAL}

See supplementary material for the detailed experimental setup of the two condensation chamber configurations, and the TRIM simulation results on Au sputtering process. Also included is the mass spectra of clusters prepared at different aggregation length.

\section{ACKNOWLEDGEMENTS}

The research leading to these results has received funding from the European Union's Seventh Framework Programme (FP7/2007-2013) under grant agreement n 607417 (Catsense) and under grant agreement $n^{\circ} 604296$ (BioGo). P.L. acknowledges the Research Foundation - Flanders (FWO) and the KU Leuven Research Fund (BOF).

\section{DATA AVAILABILITY STATEMENT}

The data that support the findings of this study are available from the corresponding author upon reasonable request. 


\section{REFERENCES}

Bich, E., Millat, J. \& Vogel, E., 1990. The viscosity and thermal conductivity of pure monatomic gases from their normal boiling point up to $5000 \mathrm{~K}$ in the limit of zero density and at 0.101325 MPa. Journal of Physical and Chemical Reference Data, 19(6), pp. 1289-1305.

Chen, Y. et al., 2018. DNA Templated Metal Nanoclusters: From Emergent Properties to Unique Applications. Accounts of Chemical Research, 51(11), pp. 2756-2763.

Chorin, A. J., 1968. Numerical solution of the Navier-Stokes equations. Mathematics of Computation, Volume 22, pp. 745-762.

Christopher, P., Xin, H., Marimuthu, A. \& Linic, S., 2012. Singular characteristics and unique chemical bond activation mechanisms of photocatalytic reactions on plasmonic nanostructures. Nature Materials, 11(12), pp. 1044-1050.

Di Vece, M., 2019. Using Nanoparticles as a Bottom-up Approach to Increase Solar Cell Efficiency:. KONA Powder and Particle Journal, Volume 36, pp. 72-87.

Elger, B. et al., 2017. Electrostatic simulation of a complete cluster deposition apparatus. Review of Scientific Instruments, 88(6), p. 063303.

Ellis, P. R. et al., 2016. The cluster beam route to model catalysts and beyond. Faraday Discuss., Volume 188, pp. 39-56.

Fischer, A., Kruk, R. \& Hahn, H., 2015. A versatile apparatus for the fine-tuned synthesis of cluster-based materials. Review of Scientific Instruments, 86(2), p. 023304.

Ganeva, M., Kashtanov, P., Smirnov, B. \& Hippler, R., 2014. Angular and velocity distribution of nano-size cluster beams in a gas flow. Vacuum, Volume 110, pp. 140-145.

Grammatikopoulos, P., Sowwan, M. \& Kioseoglou, J., 2019. Computational Modeling of Nanoparticle Coalescence. Advanced Theory and Simulations, 2(6), p. 1900013.

Grammatikopoulos, P. et al., 2016. Nanoparticle design by gas-phase synthesis. Advances in Physics: $X, 1(1)$, pp. 81-100.

Green, J. \& Paget, M. S., 2004. Bacterial redox sensors. Nature Reviews Microbiology, 2(12), pp. 954-966.

Haberland, H. et al., 1994. Filling of micron-sized contact holes with copper by energetic cluster impact. Journal of Vacuum Science \& Technology A, 12(5), pp. 2925-2930.

Hartmann, H. et al., 2012. Design and capabilities of an experimental setup based on magnetron sputtering for formation and deposition of size-selected metal clusters on ultraclean surfaces. Review of Scientific Instruments, \#jul\#, 83(7), p. 073304.

Hinze, J. O., 1975. Turbulence. s.I.:McGraw-Hill Publishing Co..

Huang, P. G., Bradshaw, P. \& Coakley, T. J., 1993. Skin friction and velocity profile family for compressible turbulentboundary layers. AIAA Journal, 31(9), pp. 1600-1604.

Johnston, R. L., 2002. Atomic and Molecular Clusters. s.I.:Taylor \& Francis. 
Kader, B., 1981. Temperature and concentration profiles in fully turbulent boundary layers. International Journal of Heat and Mass Transfer, 24(9), pp. 1541-1544.

Kashtanov, P. V., Smirnov, B. M. \& Hippler, R., 2007. Magnetron plasma and nanotechnology. Physics-Uspekhi, 50(5), pp. 455-488.

Khojasteh, M. \& Kresin, V. V., 2017. Influence of source parameters on the growth of metal nanoparticles by sputter-gas-aggregation. Applied Nanoscience, Issue 7, pp. 875-883.

Kousal, J. et al., 2013. Characterization of nanoparticle flow produced by gas aggregation source. Vacuum, Volume 96, pp. 32-38.

Kousal, J. et al., 2018. Magnetron-sputtered copper nanoparticles: lost in gas aggregation and found by in situ X-ray scattering. Nanoscale, 10(38), pp. 18275-18281.

Kumar, V. V. \& Anthony, S. P., 2016. Chapter 9 - Antimicrobial studies of metal and metal oxide nanoparticles. In: A. M. Grumezescu, ed. Surface Chemistry of Nanobiomaterials. s.I.:William Andrew Publishing, pp. 265-300.

Launder, B. \& Spalding, D., 1974. The numerical computation of turbulent flows. Computer Methods in Applied Mechanics and Engineering, 3(2), pp. 269-289.

Li, A. \& Ahmadi, G., 1992. Dispersion and Deposition of Spherical Particles from Point Sources in a Turbulent Channel Flow. Aerosol Science and Technology, 16(4), pp. 209-226.

Linic, S., Aslam, U., Boerigter, C. \& Morabito, M., 2015. Photochemical transformations on plasmonic metal nanoparticles. Nature Materials, 14(6), pp. 567-576.

Luk'yanchuk, B. et al., 2010. The Fano resonance in plasmonic nanostructures and metamaterials. Nature Materials, 9(9), pp. 707-715.

Mattei, J.-G.et al., 2019. Gas-Phase Synthesis of Trimetallic Nanoparticles. Chemistry of Materials, 31(6), pp. 2151-2163.

Mayoral, A. et al., 2018. Tuning the size, composition and structure of Au and Co50Au50 nanoparticles by high-power impulse magnetron sputtering in gas-phase synthesis. Nanotechnology, 30(6), p. 065606.

Menter, F. R., 1994. Two-equation eddy-viscosity turbulence models for engineering applications. AIAA Journal, 32(8), pp. 1598-1605.

Popok, V. N., Barke, I., Campbell, E. E. B. \& Meiwes-Broer, K.-H., 2011. Cluster-surface interaction: From soft landing to implantation. Surface Science Reports, 66(10), pp. 347-377.

Pratontep, S. et al., 2005. Size-selected cluster beam source based on radio frequency magnetron plasma sputtering and gas condensation. Review of Scientific Instruments, \#mar\#, 76(4), p. 045103.

Reynolds, W. C., 1987. Fundamentals of Turbulence for Turbulence Modeling and Simulation. s.l., s.n.

Rossnagel, S. M., 1988. Deposition and redeposition in magnetrons. Journal of Vacuum Science \& Technology A, 6(6), pp. 3049-3054. 
Sanna, G. \& Tomassetti, G., 2005. Introduction to Molecular Beams Gas Dynamics. s.I.:Imperial College Press.

Sanzone, G. et al., 2018. Ag/TiO2 nanocomposite for visible light-driven photocatalysis. Superlattices and Microstructures, Volume 123, pp. 394-402.

Schreiner, J., Schild, U., Voigt, C. \& Mauersberger, K., 1999. Focusing of Aerosols into a Particle Beam at Pressures from 10 to 150 Torr. Aerosol Science and Technology, 31(5), pp. 373-382.

Serrano-Guisan, S. et al., 2006. Enhanced magnetic field sensitivity of spin-dependent transport in cluster-assembled metallic nanostructures. Nature Materials, 5(9), pp. 730-734.

Shih, T.-H.et al., 1995. A new k-e eddy viscosity model for high reynolds number turbulent flows. Computers \& Fluids, 24(3), pp. 227-238.

Shyjumon, I. et al., 2006. Deposition of titanium/titanium oxide clusters produced by magnetron sputtering. Thin Solid Films, 500(1), pp. 41-51.

Simon, T. et al., 2014. Redox shuttle mechanism enhances photocatalytic $\mathrm{H} 2$ generation on $\mathrm{Ni}-$ decorated CdS nanorods. Nature Materials, 13(11), pp. 1013-1018.

Smirnov, B., 2012. Clusters and Small Particles: In Gases and Plasmas. s.I.:Springer New York.

Vahl, A. et al., 2017. Single target sputter deposition of alloy nanoparticles with adjustable composition via a gas aggregation cluster source. Nanotechnology, 28(17), p. 175703.

Vajda, S. et al., 2009. Subnanometre platinum clusters as highly active and selective catalysts for the oxidative dehydrogenation of propane. Nature Materials, 8(3), pp. 213-216.

Vangala, K. et al., 2012. Studying Protein and Gold Nanoparticle Interaction Using Organothiols as Molecular Probes. The Journal of Physical Chemistry C, 116(5), pp. 3645-3652.

Wallace, W. T. \& Whetten, R. L., 2000. Carbon Monoxide Adsorption on Selected Gold Clusters: Highly Size-Dependent Activity and Saturation Compositions. The Journal of Physical Chemistry B, 104(47), pp. 10964-10968.

Wegner, K., Piseri, P., Tafreshi, H. V. \& Milani, P., 2006. Cluster beam deposition: a tool for nanoscale science and technology. Journal of Physics D: Applied Physics, 39(22), pp. R439R459.

White, F. M. \& Christoph, G. H., 1971. A simple new analysis of compressible turbulent twodimensional skin friction under arbitrary conditions, University of Rhode Island: s.n.

Xirouchaki, C. \& Palmer, R. E., 2002. Pinning and implantation of size-selected metal clusters: a topical review. Vacuum, 66(2), pp. 167-173.

Zhang, L., Shao, J. \& Chen, X., 2016. CFD simulation of nozzle characteristics in a gas aggregation cluster source. Vacuum, Volume 129, pp. 105-110.

Zhang, Z. H. et al., 2009. Evidence of intrinsic ferromagnetism in individual dilute magnetic semiconducting nanostructures. Nature Nanotechnology, 4(8), pp. 523-527. 


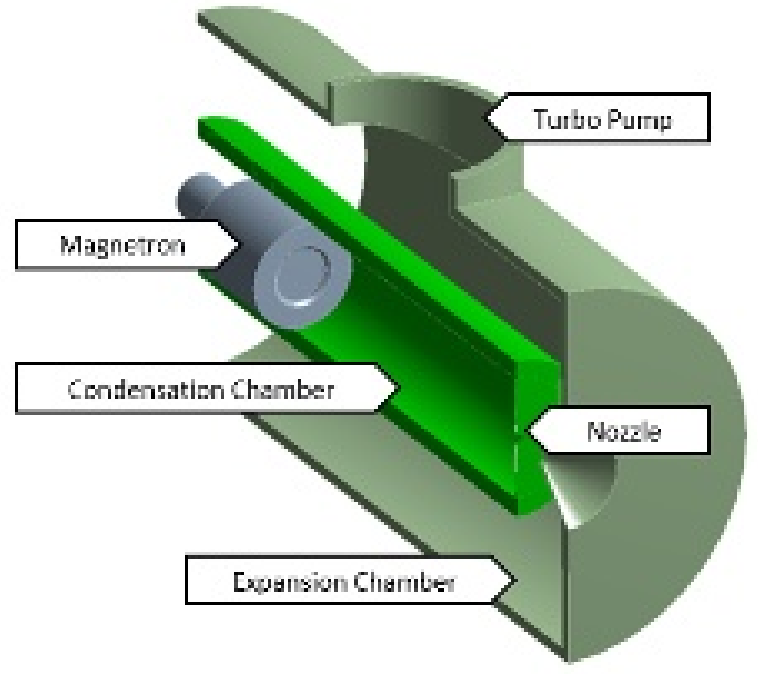




\section{Condensation chamber}

MFC

Gas flow controller
Valve

Valve

alve

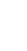




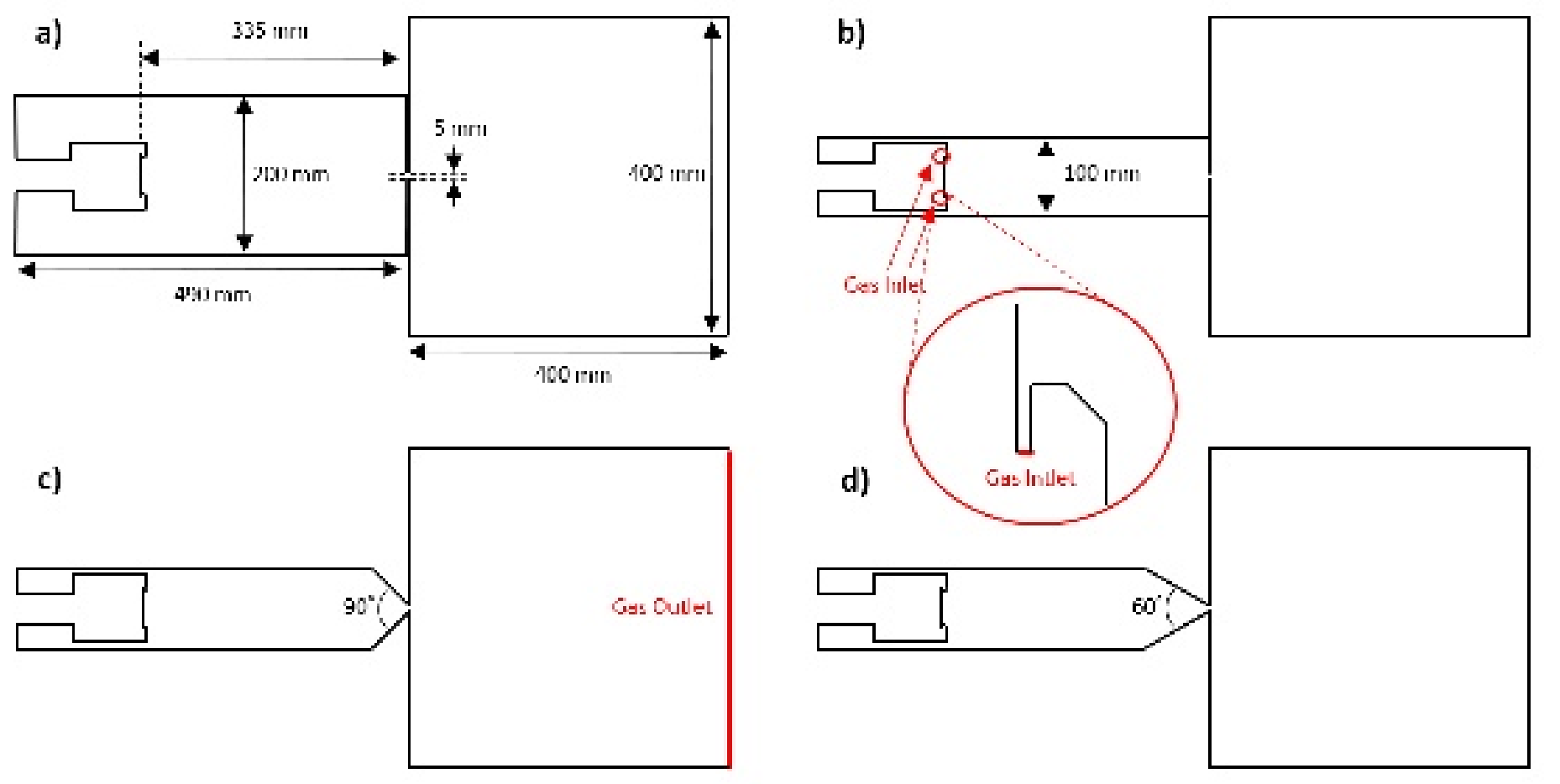




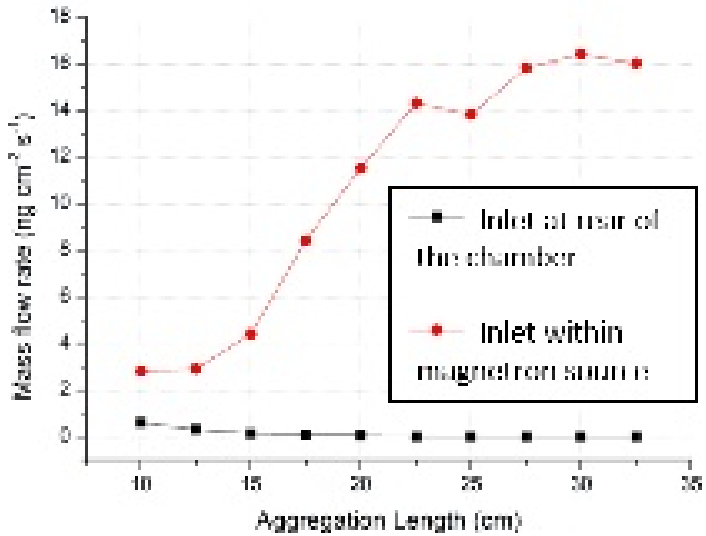




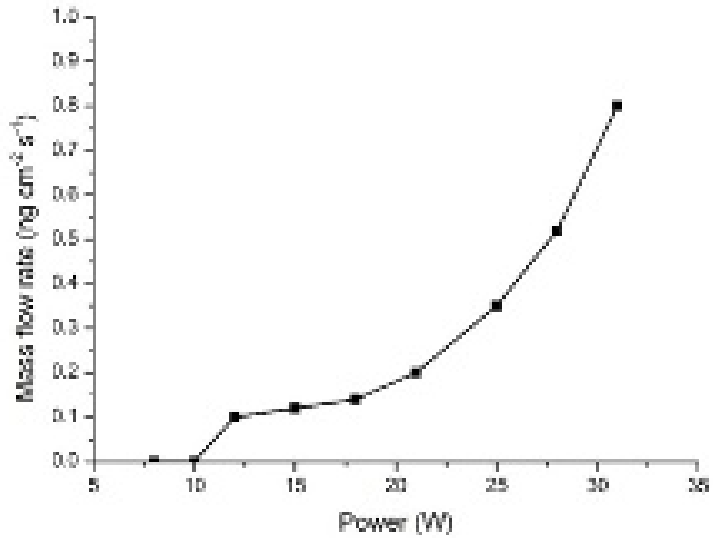




\section{Wecter}

$+0 m+600$

$100=60$ :

1.00-021

$10 \mathrm{~s}-0=2$ [n $\% \cdot 1]$

$\mathrm{men}+\mathrm{cos}$ (ni) inlet within magernon saven

\section{Magnetron}
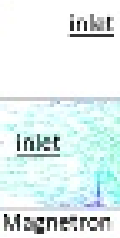

f.' Inlat at rear of the charhas 
Au atom number density

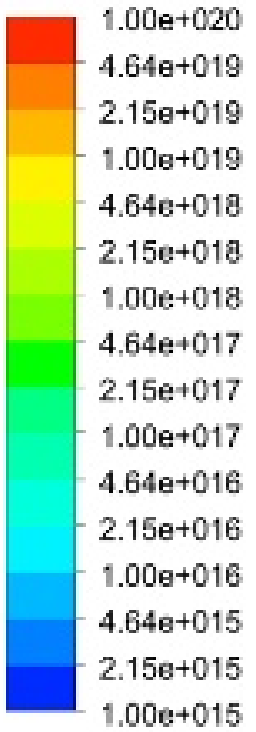

$\left[\mathrm{m}^{n-3]}\right.$

b)

Au atom number density

$1.90 \mathrm{e}+020$
$1.80 \mathrm{e}+020$
$1.70 \mathrm{e}+020$
$1.60 \mathrm{e}+020$
$1.50 \mathrm{e}+020$
$1.40 \mathrm{e}+020$
$1.30 \mathrm{e}+020$
$1.20 \mathrm{e}+020$
$1.10 \mathrm{e}+020$
$1.00 \mathrm{e}+020$
$9.00 \mathrm{e}+019$
$8.00 \mathrm{e}+019$
$7.00 \mathrm{e}+019$
$6.00 \mathrm{e}+019$
$5.00 \mathrm{e}+019$
$4.00 \mathrm{e}+019$
$3.00 \mathrm{e}+019$
$2.00 \mathrm{e}+019$
$1.00 \mathrm{e}+019$

$\left[m^{n-3}\right]$
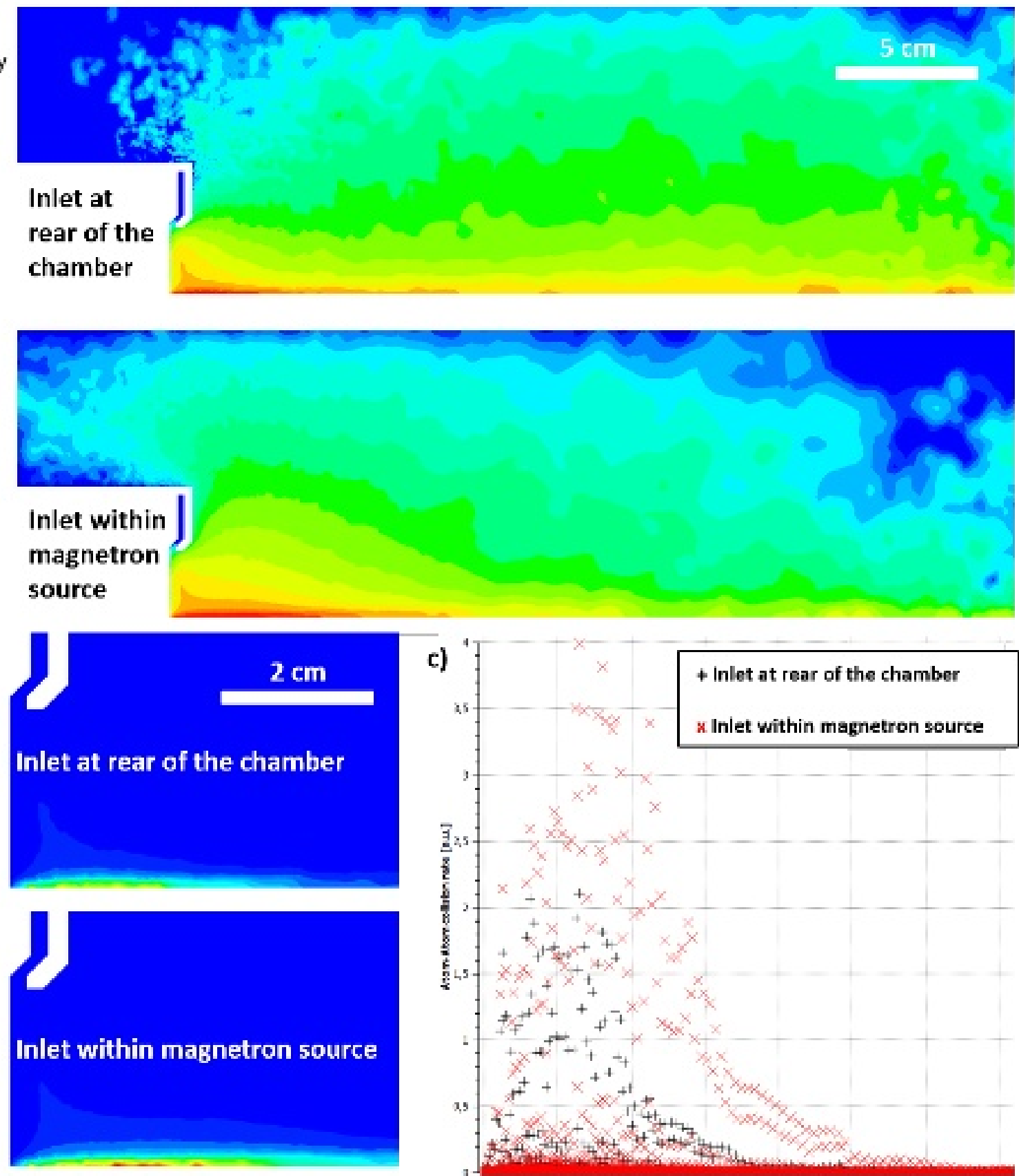


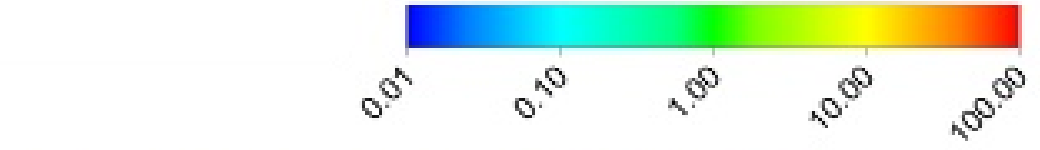

a)

b)

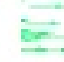

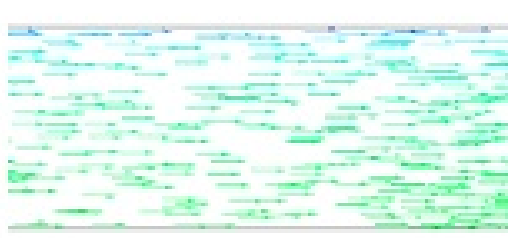

d)

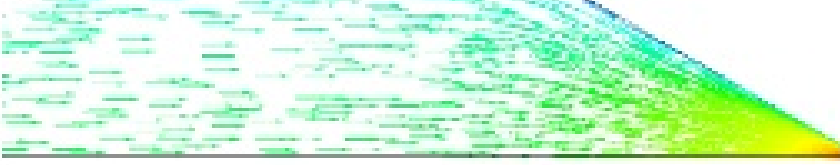



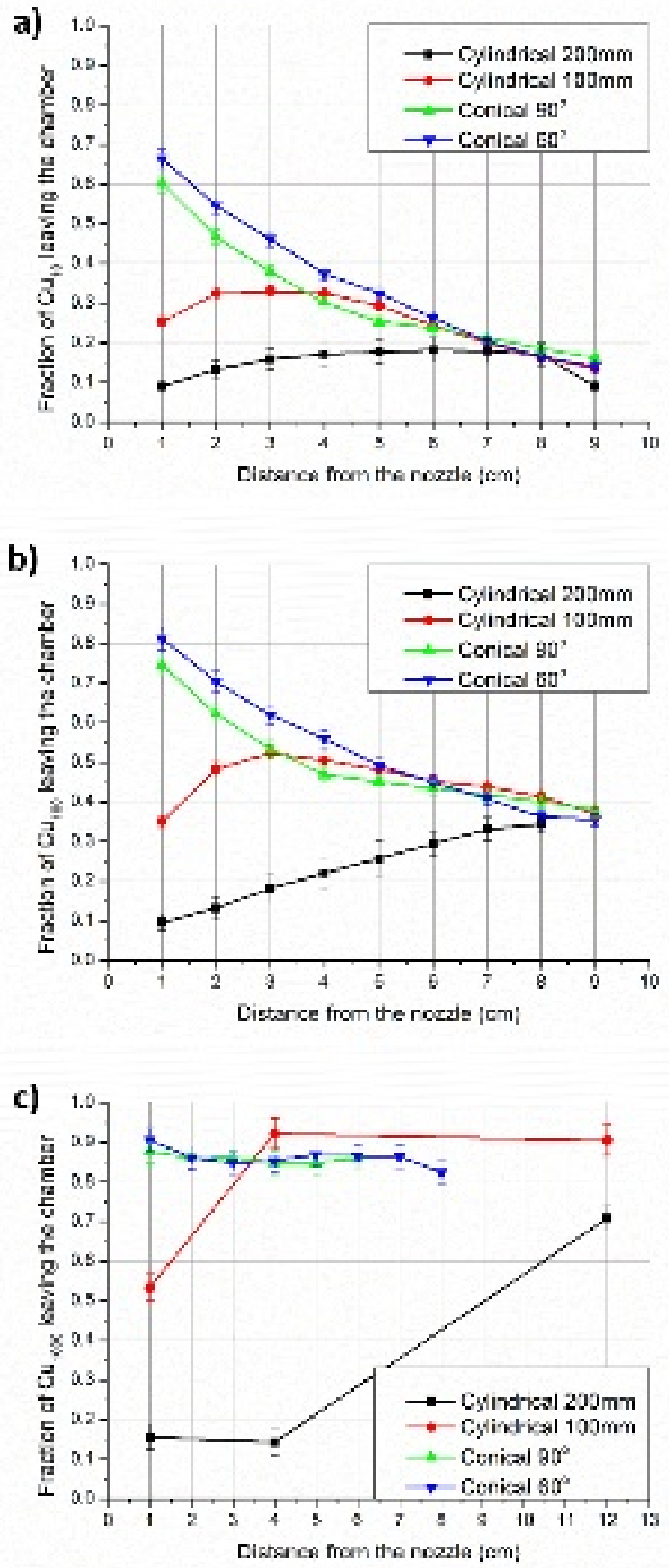


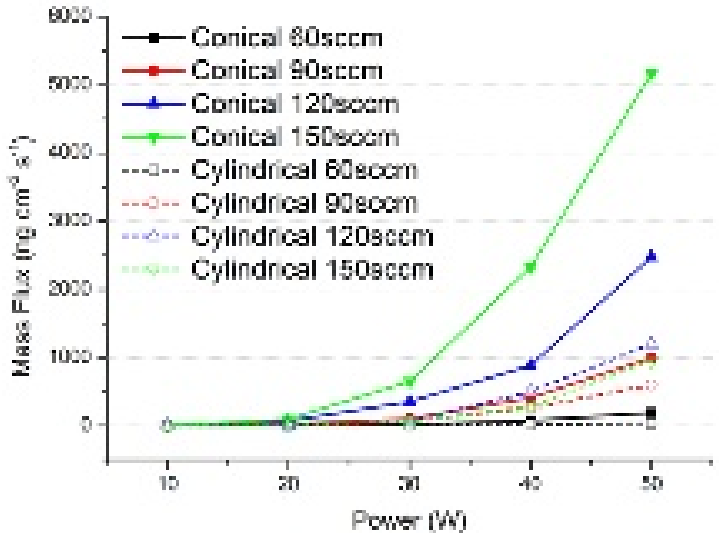

